\title{
Fine-tuning of stress-induced martensite in TRIP/TWIP Ti alloys
}

\author{
Bingnan QIAN ${ }^{1}$, Fan SUN ${ }^{1, *}$, Philippe VERMAUT ${ }^{1,2}$, Frédéric PRIMA ${ }^{1}$ \\ ${ }^{1}$ PSL Research University, Chimie ParisTech-CNRS, Institut de Recherche de Chimie Paris, F-75005 Paris, France \\ 2 Sorbonne Universities, UPMC University Paris 06, UFR926, F-75005 Paris, France
}

\begin{abstract}
$\underline{\text { Abstract }}$
Fine-tuning of stress-induced martensitic (SIM) transformation was studied in Ti-Mo based $\beta$ metastable alloys, showing combined Transformation Induced Plasticity (TRIP) and Twinning Induced Plasticity effects (TWIP) effects. The work aimed to clarify the transition and interaction between the two deformation mechanisms and their influences on the mechanical properties of Ti-Mo based alloys. Electron parameter design methods (Bo-Md and e/a ratio) were cross-used to increase the $\beta$ phase stability from near-TRIP to near-TWIP by adding third alloying elements. SIM $\alpha$ " transformation and mechanical twinning were traced by in-situ EBSD (Electron backscatter diffraction) mapping under cyclic tension. The deformation modes of $\beta$ phase exhibited significant changes when shifting its metastability via chemical composition modifications. In near-TRIP conditions (dominated by the growth of SIM $\alpha^{\prime \prime}$ in plastic regime), SIM $\alpha^{\prime \prime}$ transformation and internal twinning of martensite were the main mechanisms to accommodate the local stress-strain conditions. In near-TWIP ones (dominated by the growth of $\{332\}<113>\beta$ twinning in plastic regime), SIM $\alpha^{\prime \prime}$ was observed only at twinning interface during strain process and disappeared after stress release.
\end{abstract}

Keywords: TRIP/TWIP Ti alloys, In-situ EBSD, Stress-induced martensitic transformation, Mechanical twinning, $\beta$ metastable alloys.

*Corresponding author. E-mail: Fan.sun@,chimieparistech.psl.eu

(C) The Authors, published by EDP Sciences. This is an open access article distributed under the terms of the Creative Commons Attribution License 4.0 (http://creativecommons.org/licenses/by/4.0/). 


\section{TiMo based TRIP/TWIP alloys}

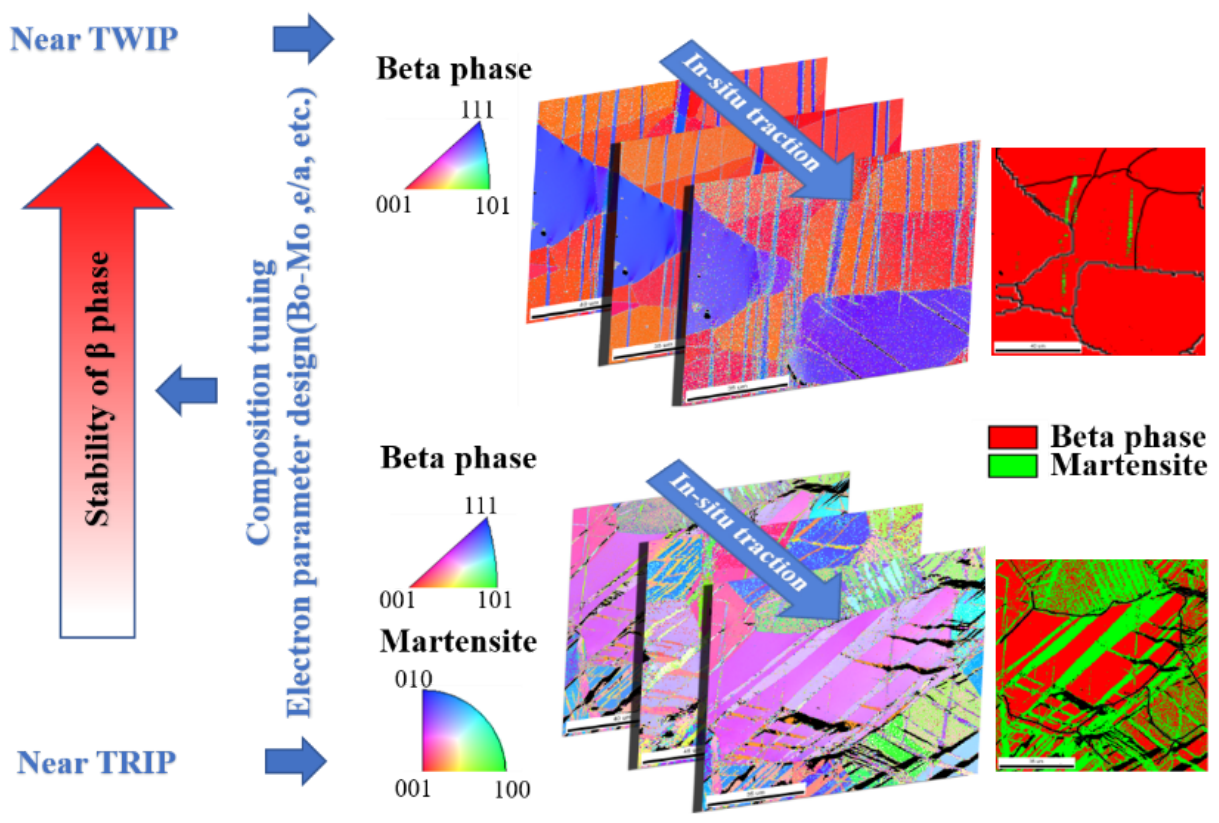

\section{Introduction}

Since the last few years, a new family of metastable $\beta$ titanium alloys with TRansformation Induced Plasticity and/or TWinning Induced Plasticity effects (denoted as TRIP/TWIP hereafter) has been developed by design based on electron parameter design methods (Bo-Md and e/a ratio). The TRIP/TWIP Ti alloys present an unprecedented combination of high strength, large ductility, and excellent work hardening rate when compared to the conventional Ti alloys [1-4]. Recent studies have reported complex deformation mechanisms mainly involving mechanical twinning ( $\{332\}$ mode, $\{112\}$ mode in $\beta$ BCC) and stress/strain-induced martensite (SIM $\alpha^{\prime \prime}, \quad$ BCC $\beta ® \alpha^{\prime \prime}$ Orthorhombic) [5, 6] The simultaneous activation of dislocations slip with the various mechanisms results in dynamic Hall-Petch effect, heterogeneous composite effect, hierarchical twin/SIM microstructure, multimodal twinning network, etc [1-7].

It is well-known that the activation of mechanical twinning and SIM closely depends on the stability of the $\beta$ phase. Martensitic transformation $\left(\beta{ }^{\circledR} \alpha^{\prime \prime}\right)$ can be activated by applying external stress in the $\beta$-metastable alloys when the chemical stability of $\beta$ phase (usually estimated by Mo-equivalent content, $\mathrm{Mo}_{\mathrm{eq}}$ wt.\%) is relatively low (a rough range of $10.5 \% £$ alloy $\mathrm{Mo}_{\mathrm{eq}} £ 13 \%$ ) [8]. Mechanical twinning can be activated when the $\beta$ stability becomes higher (roughly $12 \% £$ alloy $\mathrm{Mo}_{\mathrm{eq}} £ 15 \%$ ) [8]. The overlapping of the two ranges allows the simultaneous occurrence of TRIP and TWIP effects in the same alloy. Furthermore, a continuous transition of deformation mechanisms, from near-TRIP to near-TWIP, can be expected by increasing the chemical stability of a TRIP/TWIP alloy.

$\mathrm{Zr}$ has been found to be a neutral element (in Ti-Zr system) or weak $\beta$-stabilizing element (in Ti-Nb-Zr system) [9, 10]. Previous studies reported that the nature of $\mathrm{Zr}$ in a Ti-M-Zr system $(\mathrm{M}=\beta$-stabilizer alloying element $)$ strongly depends on the $\beta$-stability and concentration of $\mathrm{M}[9]$. The electron parameter (Bo-Md and e/a ratio) design methods suggest that the addition of $\mathrm{Zr}$ could 
shift gently TRIP/TWIP alloys towards TWIP region [11]. In order to study the effect of $\mathrm{Zr}$ addition on the TRIP to TWIP transition in Ti-Mo system, a comparative investigation is performed between the typical TRIP/TWIP Ti-12Mo (wt. \%) [1, 12] alloy and two Ti-12Mo-xZr (x = 5, 10) (wt. \%) alloys designed by Bo-Md method.

Electron Backscattered Diffraction (EBSD) characterizations coupled with in-situ tensile deformation are employed to study the effects of $\mathrm{Zr}$ addition on the deformation mechanisms. The in-situ method is used to reveal the formation, development, and reversion of martensite/twin during the tensile loading and unloading processes.

\section{Experimental}

The $200 \mathrm{~g}$ ingots with nominal compositions of $\mathrm{Ti}-12 \mathrm{Mo}-\mathrm{xZr}(\mathrm{x}=0,5,10)$ were fabricated by vacuum arc-melting using pure Ti, Mo and $\mathrm{Zr}$ metals (purity $>99.9 \mathrm{wt} . \%$ ). Each ingot was remelted for five times and homogenized at $1373 \mathrm{~K}$ for $72 \mathrm{ks}$ under a high vacuum of $10^{-4} \mathrm{~Pa}$, followed by water quenching. The as-quenched ingots were cut to a billet of $10 \mathrm{~mm}$ in thickness $65 \mathrm{~mm}$ in diameter. The billet was cold-rolled to $0.5 \mathrm{~mm}$ in thickness (reduction rate of 95\%). Subsize tensile samples (ASTM E8M) and insitu samples (L40mm, W2 mm, T0.5mm) were cut from the as-rolled sheet along the rolling direction. The samples were solutiontreated (ST) at $1173 \mathrm{~K}$ for $1.8 \mathrm{ks}$ under high vacuum then water quenched.

The uniaxial tensile tests were performed with a constant strain rate of $10^{-3} \mathrm{~s}^{-1}$ using INSTRON 5982 machine. The in-situ samples were polished to mirror-like surface finishing. In-situ EBSD scans were performed using a field emission gun scanning electron microscope (FEG-SEM Zeiss LEO1530 with a high-speed Nordif UF-1100 EBSD camera) operating at 20kV. The indexation of EBSD raw data was performed by TSL OIM Analysis Software. The EBSD scans were performed by holding the tensile strain before loading, during loading and after unloading on a Proxima 100-Micromecha in-situ tensile test machine.

\section{Results and discussion}

ST samples of Ti-12Mo-xZr exhibit typical recrystallized $\beta$ grains. The phase constitutions of the ST Ti-Mo-Zr samples were identified to be a fully $\beta$ matrix with quenched-in athermal omega phase (nanometric $\omega_{\text {ath }}$ ) similar to Ti-12Mo $[1,12](X R D$ and TEM results are not shown here).

The tensile properties of the 3 alloys are listed in table 1. The YS and UTS strongly increase with the Zr content, whereas the ductility reduces. The strengthening might be due to solution strengthening effect of $\mathrm{Zr}$ addition. The reduction of ductility could be caused by many reasons, one of them could probably be the change of deformation mechanism.

Table 1 Phase constitutions, average grain size, and tensile properties of Ti-12Mo (wt. \%) alloy and Ti12MoxZr (x= 5, 10) (wt. \%) alloys 


\section{Ti12Mo}

Phases

YS (MPa)

UTS (MPa)

$\delta 1 / 1(\%)$
Ti12Mo5Zr

Ti12Mo10Zr

$\beta+\omega_{\text {ath }} \quad \beta+\omega_{\text {ath }}$

565

740

929

1040

921

30.4

24.3

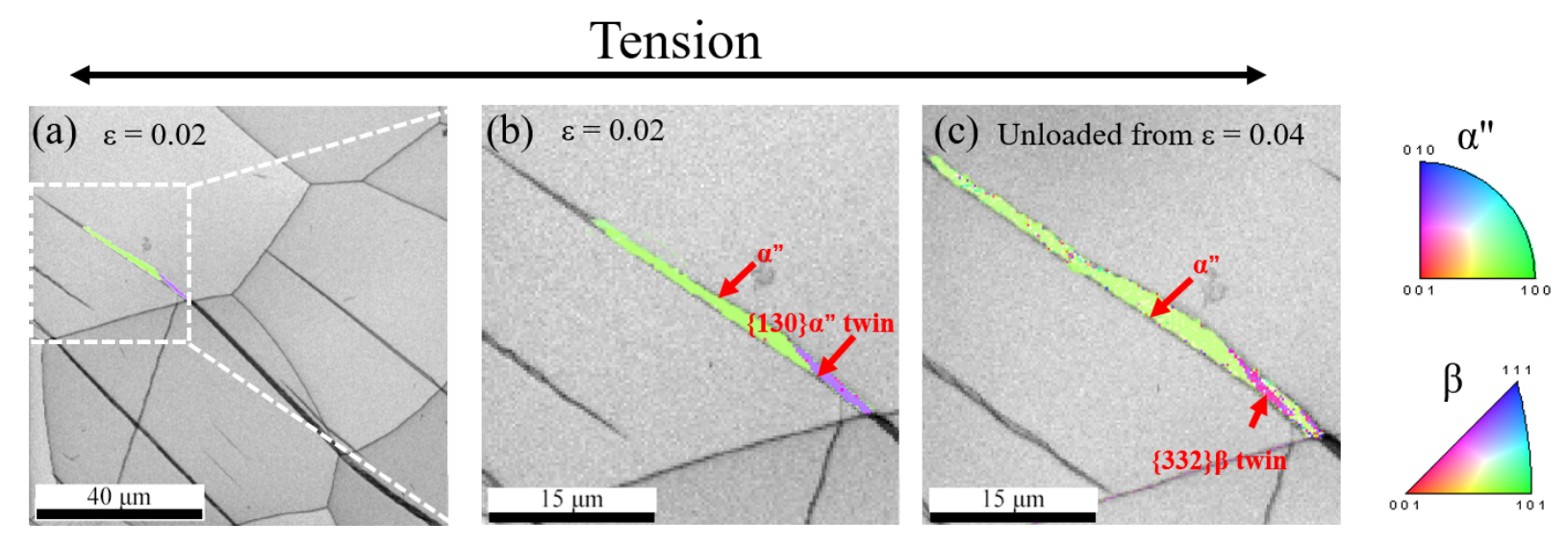

Fig. 1 Inverse pole figure (IPF) + image quality (IQ) map of Ti-12Mo at (a) in-situ loading to $\varepsilon=0.02$, (b) SIM $\alpha^{\prime \prime}$ and its $\{130\}<310>\alpha^{\prime \prime}$ twin of the deformation band at in-situ $\varepsilon=0.02$, and (c) SIM $\alpha^{\prime \prime}$ and $\{332\}<113>_{\beta}$ twin occurred in the same band after unloading from $\varepsilon$ $=0.04$.

Figure 1 shows the EBSD analysis of Ti-12Mo sample tracing the microstructure evolution at different strains under loading at $\varepsilon=$ 0.02 , and after unloading from $\varepsilon=0.04$ ). The Ti-12Mo showed typical TRIP/TWIP microstructure) $[\mathbf{1}, \mathbf{1 2}]$ at $\varepsilon=0.02$ corresponding to the end of elastic-plastic transition. The in-situ EBSD observation was focused on a SIM band (zoom box is white square in fig. 1a). The Fig. 1a and 1b show that two variants of SIM $\alpha$ " phase ("green" variant and "purple" variant) occurred in the same band after $\varepsilon=0.02$ deformation, the orientation relationship between the "green" SIM $\alpha$ " and surrounding $\beta$ matrix is in agreement with the classical crystallographic relationship $\{211\} \beta / /\{110\} \alpha^{\prime \prime}$ of the $\beta{ }^{\circledR} \alpha^{\prime \prime}$ martensitic transformation. It might suggest that the "green" SIM $\alpha$ " is the primary product originated from direct $\beta{ }^{\circledR} \alpha$ " transformation [13]. However, no such relationship can be found between the "purple" $\alpha$ " variant and $\beta$ matrix. In contrast, a classical $\{130\}<310>^{\prime \prime}$ twin relationship was found between the "green" and "purple" $\alpha$ " variants. This suggests that the $\{130\}<310>\alpha^{\prime}$ mechanical twinning occurred in $\alpha^{\prime \prime}$ SIM during the straining. Such twinning mode has already been reported by Bertrand et al. [14] in deformed Ti$25 \mathrm{Ta}-20 \mathrm{Nb}$ wt. $\%$ alloy. After loading to $\varepsilon=0.04$ and fully unloaded, the "purple" $\alpha$ " variant disappeared by transforming partially back to "green" $\alpha$ " variant and partially reversed to $\beta$ phase ("pink" $\beta$ phase in fig. 1c). This "pink" $\beta$ phase exhibits a $\{130\}$ $<310>_{\alpha^{\prime \prime}}$ twin type misorientation with the $\beta$ surrounding matrix (fig. 1c). This phenomenon has been reported by Castany et al. [5] in Ti-Nb system. In the present case, one can considered that $\{332\}<113>_{\beta}$ twins are thus not formed directly from the $\beta$ phase but are the result of the reversion of $\{130\}<310>\alpha^{\prime \prime}$ parent twins occurring in $\alpha^{\prime \prime}$ SIM during unloading. 


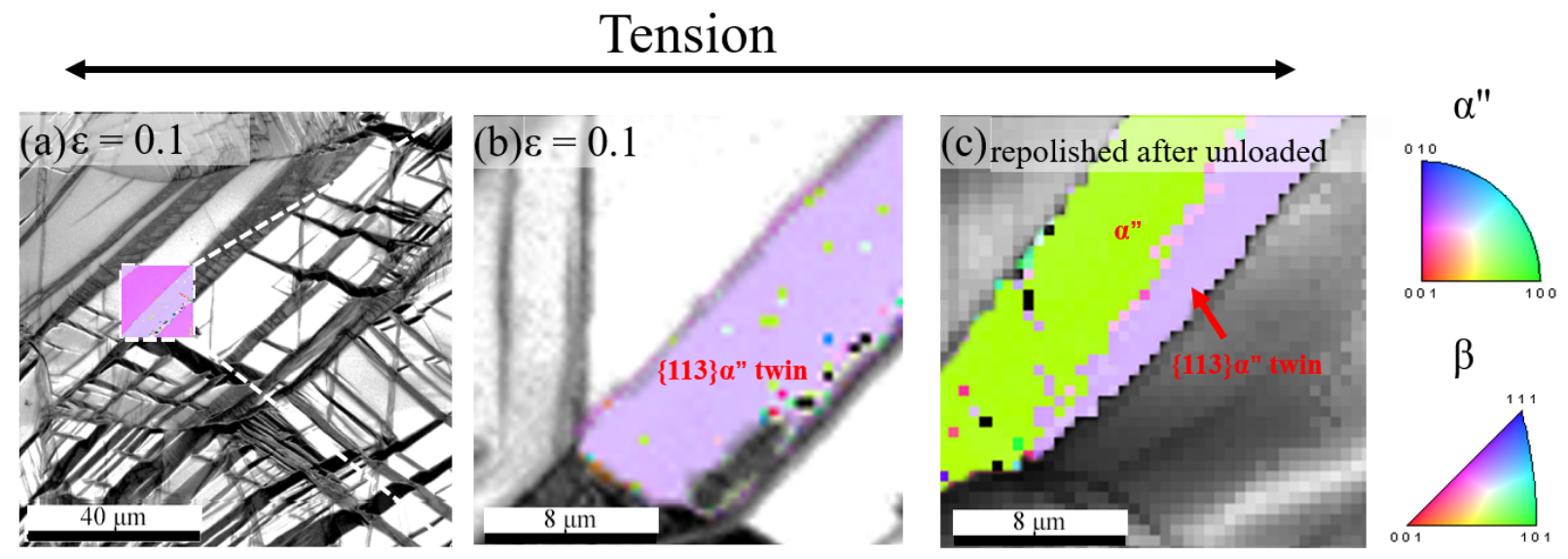

Fig. 2 Inverse pole figure (IPF) + image quality (IQ) map of Ti-12Mo-5Zr at (a) in-situ loaded and held at $\varepsilon=0.1$, (b) $\{130\}<310>\alpha^{\prime \prime}$ twin band zoomed at white square at $\varepsilon=0.1$, and (c) SIM $\alpha^{\prime \prime}$ and $\{130\}<310>\alpha^{\prime \prime}$ twin in thus band repolished after unloaded from $\varepsilon=0.1$.

In-situ EBSD maps of Ti-12Mo-5Zr are shown in figure 2. The sample presents a deformation microstructure similar to Ti-12Mo at $\varepsilon=0.02$ and 0.04 . At a higher deformation level $(\varepsilon=0.1)$ a high density of deformation bands is visible in figure $2 \mathrm{a}$. A primary martensite band (white square fig. 2a zoomed in fig. 2b) was thus followed by extending the in-situ loading up to $\varepsilon=0.1$. The thickening of the $\alpha^{\prime \prime}$ martensite band was recorded up to about $10 \mu \mathrm{m}$ and remained single variant ("purple" $\alpha$ " variant). The crystallographic relationship analysis between this "purple" $\alpha$ " and its surrounding b matrix, again does not satisfy the classical b/ $\alpha^{\prime \prime}$ orientation relationships. It can be noticed that the image quality of the band at $\varepsilon=0.1$ (Fig. 2a) was affected due to important surface relief. In order to improve the EBSD map quality, a fine re-polishing (depth $<5 \mu \mathrm{m}$ ) was applied to the unloaded sample to remove the surface relief. The EBSD map of the same band after re-polishing (Fig. 2c) presented two variants, a "green" and a "purple" variant, in which the "purple" variant was the same variant observed under load at e=0.1 (Fig. 2a and 2b). The crystallographic relationships among the "green" $\alpha$ " variant, "purple" $\alpha$ " variant and $\beta$ matrix were identified as:

i. "green" and "purple" $\alpha$ " variants yielded $\{130\}<310>_{\alpha \text { " }}$ twin relationships;

ii. "green" $\alpha$ " and $\beta$ matrix yielded classical $\beta \rightarrow \alpha$ " martensitic transformation relationships;

iii. "purple" $\alpha$ " and $\beta$ matrix did not yield classical $\beta \otimes \alpha "$ martensitic transformation relationships.

This analysis suggests again that, during loading, the $\mathrm{b}$ phase transformed into $\alpha^{\prime \prime}$ martensite which immediately mechanically twinned with the $\{130\}<310>_{\alpha^{\prime \prime}}$ mode. This situation is very similar to the above Ti-12Mo except that the martensite did not reverse into $b$ phase when the sample was unloaded. The observed deformation mechanism of Ti-12Mo-5Zr was then close to that of Ti-12Mo at the early stage of the plastic regime. Addition of 5wt.\% Zr did not strongly affect the occurrence of the $\alpha$ " SIM and its mechanical twinning. This suggests that $5 \mathrm{wt} . \% \mathrm{Zr}$ introduced noticeable solid solution strengthening but did not change significantly the $\beta$ stability of Ti-12Mo. 


\section{Tension}
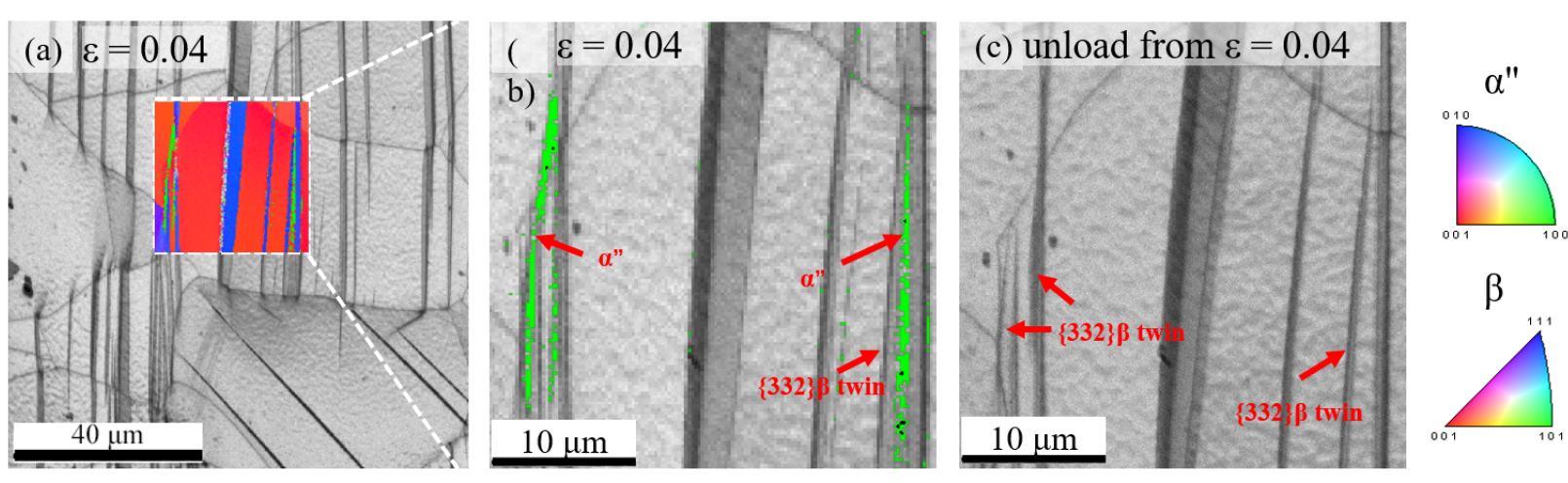

Fig. 3 Inverse pole figure (IPF) + image quality (IQ) map of Ti12Mo10Zr at (a) in-situ loaded and held at $\varepsilon=0.04$, (b) the SIM $\alpha^{\prime \prime}$ is highlighted using its IPF at $\varepsilon=0.04$, and (c) unloaded from $\varepsilon=0.04$, the SIM $\alpha^{\prime \prime}$ is fully reversed to beta phase (matrix).

In contrast, the deformation mechanisms appeared to be significantly modified by $10 \mathrm{wt} \% \mathrm{Zr}$ addition. Figure 3 shows in-situ EBSD maps of deformed Ti12Mo10Zr sample.

Almost all the observed major deformation bands were $\{332\}<113>_{\beta}$ from yielding to $\varepsilon=0.04$. The $\{332\}<113>\beta$ ("blue" variant in Fig. 3a) became the dominant deformation mechanism in the Ti-12Mo-10Zr sample. A minor volume fraction of $\alpha^{\prime \prime}$ SIM ("green" variant in Fig. 3a and 3b) was observed, always connected to $\beta$ twin. In order to figure out if the $\alpha$ " SIM is from b matrix or $\{332\}<113>_{\beta}$ twin, the orientation relationship analysis has been carried out. The results showed that the $\alpha^{\prime \prime}$ SIM yielded the classical $\beta \circledR \alpha^{\prime \prime}$ martensitic transformation relationships, whilst no classical relationships were found between the $\alpha^{\prime \prime}$ SIM and the adjacent $\{332\}<113>_{\beta}$ twin. This suggested that the $\alpha^{\prime \prime}$ SIM transformed from $\beta$ matrix but nucleated at $\{332\}<113>_{\beta}$ twin interface. When unloaded, the $\alpha^{\prime \prime}$ SIM fully transformed back to the $\beta$ matrix (Fig. 3c), suggesting that its formation probably acts as a relaxation mechanism to accommodate local stress which happened usually near twin interfaces and their intersections.

The fact that the primary deformation mechanism changes from $\alpha^{\prime \prime}$ SIM to $\{332\}<113>\beta$ mechanical twinning of the $\beta$ phase when the $\mathrm{Zr}$ amount increases, suggests that this alloying element increases the critical shear stress of SIM $\alpha^{\prime \prime}$, confirming the $\beta$ stabilizing character of this element in Ti-Mo alloys. This stabilization of the $\beta$ phase limits the formation of $\alpha^{\prime \prime}$ SIM to high-stress level volumes. This could explain the observed ductility reduction of Ti-12Mo-10Zr because of the partial suppression of relaxational effect of the $\alpha^{\prime \prime}$ SIM.

According to the comparative study of the samples, two effects can be noticed of the Zr addition to the SIM in TRIP/TWIP Ti12Mo:

i. no obvious modification up to $5 \mathrm{wt} . \% \mathrm{Zr}$ addition;

ii. significant reduction of SIM with $10 \mathrm{wt} . \% \mathrm{Zr}$ addition. 
$\mathrm{Zr}$ started to increase the $\beta$ phase stability of Ti-12Mo when $>5 \mathrm{wt} . \%$, leading to the shift of the deformation mechanism from TRIP/TWIP to TWIP-dominant. Therefore, a fine-tuning of the SIM could probably be achieved by controlling $\mathrm{Zr}$ content in metastable Ti-Mo-Zr system.

\section{Conclusion}

Several effects of $\mathrm{Zr}$ additions were observed on the mechanical properties and deformation mechanisms of Ti-12Mo-xZr alloys. A noticeable solid-solution strengthening effect was obtained with both $5 \%$ and $10 \% \mathrm{Zr}$ additions. A shift of deformation mechanism from combined TRIP/TWIP to TWIP-dominant was observed when $\mathrm{Zr}$ content is increased from 5\% to $10 \%$. The fine-tuning of the volume fraction of SIM can be achieved in the 5\% - 10\% $\mathrm{Zr}$ range, in which $\mathrm{Zr}$ acts as a b-stabilizer in Ti-12Mo. Thanks to insitu tensile tests connected to EBSD mapping, the first evidence of $\{130\}<310>_{\alpha^{\prime \prime}}$ twins reverse transformation to $\{332\}<113>_{\beta}$ twin was observed in Ti-12Mo during an in-situ loading-unloading cycle. New insights about the SIM-Twinning interactions were revealed in the complex TRIP/TWIP microstructure evolution.

\section{Acknowledgments}

The authors would like to acknowledge Dr. Jinyong ZHANG and his research team at China University of Mining and Technology for ingot fabrications and raw material characterizations. This work is supported in part by the scholarship from China Scholarship Council (CSC) under the Grant CSC201806240024.

\section{$\underline{\text { References }}$}

[1] F. Sun, J.Y. Zhang, M. Marteleur, T. Gloriant, P. Vermaut, D. Laillé, P. Castany, C. Curfs, P.J. Jacques, F. Prima, Acta Mater. 61 (2013) 6406-6417.

[2] F. Sun, J.Y. Zhang, M. Marteleur, C. Brozek, E.F. Rauch, M. Veron, P. Vermaut, P.J. Jacques, F. Prima, Scripta Mater. 94 (2015) $17-20$.

[3] H. Liu, M. Niinomi, M. Nakai, K. Cho, Scripta Mater. 82 (2014) 29-32.

[4] J. Gao, Y. Huang, D. Guan, A.J. Knowles, L. Ma, D. Dye, W.M. Rainforth, Acta Mater. 152 (2018) 301-314.

[5] P. Castany, Y. Yang, E. Bertrand, T. Gloriant, Phys. Rev. Let. 117 (2016) 1-6.

[6] E. Bertrand, P. Castany, I. Péron, T. Gloriant, Scripta Mater. 64 (2011) 1110-1113.

[7] M. Ahmed, D. Wexler, G. Casillas, D. G. Savvakin, E.V. Pereloma, Acta Mater. 104 (2016) 190-200.

[8] I. Weiss, S. L. Semiatin, Mat. Sci. and Eng. A 243 (1998) 46-65. 
[9] J.Y. Zhang, F. Sun, Y.L. Hao, N. Gozdecki, E. Lebrun, P. Vermaut, R. Portier, T. Gloriant, P. Laheurte, F. Prima, Mat. Sci. and Eng. A 563 (2013) 78-85.

[10] M. Abdel-Hady, H. Fuwa, K. Hinoshita, H. Kimura, Y. Shinzato, M. Morinaga, Scripta Mater. 57 (2007) $1000-1003$.

[11] M. Abdel-Hady, K. Hinoshita, M. Morinaga, Scripta Mater. 55 (2006) 477-480.

[12] M. Marteleur, F Sun, T. Gloriant, P. Vermaut, P. J. Jacques, F. Prima, Scripta Mater. 66 (2012) 749-752.

[13] H.Y. Kim, Y. Ikehara, J.I. Kim, H. Hosoda, S. Miyazaki, Acta Mater. 54 (2006) 2419-2429.

[14] E. Bertrand, P. Castany, Y. Yang, E. Menou, T. Gloriant, Acta Mater. 105 (2016) 94-103. 